\title{
AN EXACT FORMULA FOR THE MUTUAL INDUCTANCE OF COAXIAL SOLENOIDS.
}

\author{
By Louis Cohen.
}

In his paper on Absolute Standards of Inductance, Dr. J. G. Coffin ${ }^{1}$ gave, without demonstration, an unpublished formula of Kirchhoff, for the mutual inductance of two symmetrically placed coaxial solenoids, with the remark that this formula is an exact one and very valuable. Although the calculation of inductance by this formula involves a great deal of labor, yet it seemed desirable to calculate by it the mutual inductances of some solenoids and compare the results with the values obtained by other formulæ which are only approximate, and thus get an estimate of the degree of approximation of the other formulæ. The results obtained, however, were disappointing, the values of the mutual inductance by this formula being absurd for every case calculated, thus proving the formula to be seriously in error. Feeling quite certain that Kirchhoff deduced a correct formula, which was probably corrupted in passing through several hands, Professor Rosa asked me to undertake to deduce the correct expression, and so ascertain, if possible, just where Kirchhoff's formula as given by Coffin is wrong.

Himsted $t^{2}$ in his paper on the determination of the ohm gave an exact formula for the mutual inductance of two coaxial coils, although he indicated but very briefly the deduction of this formula. It occurred to me that the formula may be modified so as to give the inductance of solenoids, and this in fact proved to be the case. Before proceeding, however, with the transformation of Himstedt's formula, it will be desirable to give a complete deduction of the formula, since Himstedt does not give the full deduction and the result is of considerable importance.

\footnotetext{
${ }^{1}$ This Bulletin, 2, p. I25; 1906.

${ }^{2}$ Himstedt, Pogg. Ann. 28, p. 338; 1886.
} 
The mutual inductance between two circular currents is:

$$
M=\iint \frac{\cos \epsilon d s d s_{1}}{r}
$$

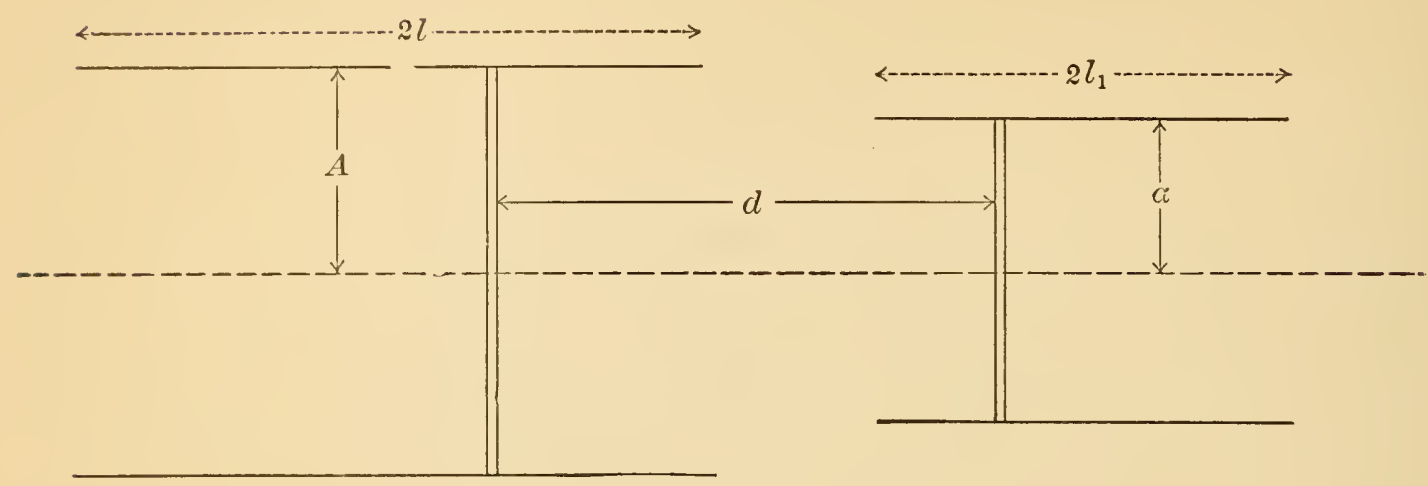

-ig. 1.

If the two circles are parallel and at a distance $d$ from each other, then

$$
\begin{aligned}
& r^{2}=A^{2}+a^{2}+d^{2}-2 A a \cos \left(\phi-\phi_{1}\right) \\
& \epsilon=\phi-\phi_{1}, d s=a d \phi, d s_{1}=A d \phi_{1}
\end{aligned}
$$

$A$ and $a$ are the radii of the two circles and therefore,

$$
M=\int_{0}^{\mathrm{e}_{\pi \pi}} \int_{2^{2 \pi}} \frac{A a \cos \left(\phi-\phi_{1}\right) d \phi d \phi_{1}}{\sqrt{A^{2}+a^{2}+d^{2}-2 A a \cos \left(\phi-\phi_{1}\right)}}
$$

which can be easily put in the following forn:

$$
M=4 \pi \int_{0}^{\pi} \frac{A a \cos \psi d \psi}{\sqrt{A^{2}+a^{2}+d^{2}-2 A a \cos \psi}}
$$

If $2 l$ and $2 l_{1}$ are the respective lengths of the two coils, $d$ the distance between the centers of the two coils, $x_{1}$ and $x_{2}$ the distances of the centers of the coils from the origin, then

$$
d=x_{1}-x_{3}
$$


and the mutual inductance of the two coils will be given by the following expression:

$$
\begin{aligned}
M & =n n^{\prime} \int_{d-\infty}^{d+l} \int_{-l}^{l} M d x d x_{1} \\
& =4 \pi n n^{\prime} \int_{d-l}^{d+l} \int_{-l}^{l} \int_{0}^{\pi} \frac{A a \cos \psi d \psi d x_{1} d x_{2}}{\sqrt{A^{2}+a^{2}+\left(x_{1}-x_{2}\right)^{2}+2 A a \cos \psi}}
\end{aligned}
$$

$n$ and $n^{\prime}$ are the number of turns per unit length of the two coils. By partial integration with respect to $\psi$ equation (3) will reduce to the following:

$$
\begin{aligned}
M= & \left.4 \pi n n^{\prime} \int_{d-l}^{a+l} \int_{-l}^{l} \frac{A a \sin \psi d x_{1} d x_{2}}{\sqrt{A^{2}+a^{2}+\left(x_{1}-x_{2}\right)^{2}-2 A a \cos \psi}}\right]_{0}^{\pi} \\
& +4 \pi n n^{\prime} \int_{d-l}^{a+l} \int_{-l}^{l} \int_{0}^{\pi} \frac{(A a)^{2} \sin ^{2} \psi d \psi d x_{1} d x_{2}}{\left[A^{2}+a^{2}+\left(x_{1}-x_{2}\right)^{2}-2 A a \cos \psi\right]^{\frac{3}{2}}} \\
= & 4 \pi m n^{\prime} \int_{d-l}^{d+l} \int_{-l}^{l} \int_{0}^{\pi} \frac{(A a)^{2} \sin ^{2} \psi d \psi d x_{1} d x_{2}}{\left[A^{2}+a^{2}+\left(x_{1}-x_{2}\right)^{2}-2 A a \cos \psi\right]^{\frac{3}{2}}}
\end{aligned}
$$

Integrating twice with respect to $x_{1}$ and $x_{2}$ and substituting the limits we get:

$$
\begin{aligned}
& M=4 \pi n n^{\prime} \int_{0}^{\pi} \frac{(A a)^{2} \sin ^{2} \psi}{A^{2}+a^{2}-2 A a \cos \psi}\left\{\sqrt{A^{2}+a^{2}+\left(d+l+l_{1}\right)^{2}-2 A a \cos \psi}\right. \\
& -\sqrt{A^{2}+a^{2}+\left(d+l_{1}-l\right)^{2}-2 A a \cos \psi} \\
& -\sqrt{A^{2}+a^{2}+\left(d+l-l_{1}\right)^{2}-2 A a \cos \psi} \\
& \left.+\sqrt{A^{2}+a^{2}+\left(d-l-l_{1}\right)^{2}-2 A a \cos \psi}\right\}
\end{aligned}
$$

When the two cuils are concentric, that is $d=0$, equation (5) will reduce to the following: 


$$
\begin{gathered}
M=4 \pi n n^{\prime} \int_{0}^{\pi} \frac{2(A a)^{2} \sin ^{2} \psi}{A^{2}+a^{2}-2 A a \cos \psi}\left\{\sqrt{A^{2}+a^{2}+c^{2}-2 A a \cos \psi}\right. \\
\left.-\sqrt{A^{2}+a^{2}+c^{2}{ }_{1}-2 A a \cos \psi}\right\} d \psi
\end{gathered}
$$

where

$$
\begin{gathered}
c=l+l_{1}, c_{1}=l-l_{1} \\
M=4 \pi n n^{\prime}\left(V-V_{1}\right)
\end{gathered}
$$

where

$$
V=\int_{0}^{\pi} \frac{2(A a)^{2} \sin ^{2} \psi \sqrt{A^{2}+a^{2}+c^{2}-2 A a \cos \psi}}{A^{2}+a^{2}-2 A a \cos \psi} d \psi
$$

and $V_{1}$ is obtained from $V$ by putting $c_{1}$ in place of $c$. The evaluation of $V$ can be obtained in the following manner:

Put

$$
\phi=2 \psi
$$

then

$$
\begin{aligned}
& \frac{V}{8(A a)^{2}}= \\
& \int_{0}^{\pi} \frac{\sin ^{2} \phi \cos ^{2} \phi\left(A^{2}+a^{2}+c^{2}-2 A a+4 A a \sin ^{2} \phi\right)}{\left(A^{2}+a^{2}-2 A a+4 A a \sin ^{2} \phi\right) \sqrt{A^{2}+a^{2}+c^{2}-2 A a+4 A a \sin ^{2} \phi}} d \phi
\end{aligned}
$$

Now make the substitution $\cos \phi=x$ then,

$$
\begin{aligned}
V & =8(A a)^{2} \int_{0}^{1} \frac{x^{2}\left(\mathrm{I}-x^{2}\right)\left\{(A+a)^{2}+c^{2}-4 A a x^{2}\right\} d x}{\left\{(A+a)^{2}-4 A a x^{2}\right\} \sqrt{(A+a)^{2}+c^{2}-4 A a x^{2} \sqrt{1-x^{2}}}} \\
& =\frac{8(A a)^{2} w}{(A+a)^{2}} \int_{0}^{1} \frac{x^{2}-\left(\mathrm{I}+\ddot{r}^{2}\right) x^{4}+k^{2} x^{6}}{\left(\mathrm{I}+n x^{2}\right) \sqrt{\left(\mathrm{I}-k^{2} x^{2}\right)\left(\mathrm{I}-x^{2}\right)}} d x
\end{aligned}
$$

where

$$
k^{2}=\frac{4 A a}{(A+\alpha)^{2}+c^{2}}, n=\frac{-4 A a}{(A+a)^{2}}, \pi e^{2}=(A+\alpha)^{2}+c^{8}
$$




$$
\begin{aligned}
& V=\frac{8(A \alpha)^{2} w}{(A+\alpha)^{2}}\left[\frac{\mathrm{I}}{n} \int_{0}^{1} \frac{\mathrm{I}+n x^{2}-\mathrm{I}}{\left(\mathrm{I}+n x^{2}\right) \sqrt{\left(\mathrm{I}-k^{2} x^{2}\right)\left(\mathrm{I}-x^{2}\right)}} d x\right. \\
& +\frac{\mathrm{I}+k^{2}}{n^{2}} \int_{0}^{1} \frac{\mathrm{I}-n^{2} x^{4}-\mathrm{I}}{\left(\mathrm{I}+n x^{2}\right) \sqrt{\left(\mathrm{I}-k^{2} x^{2}\right)\left(\mathrm{I}-x^{2}\right)}} d x \\
& \left.+\frac{k^{2}}{n^{3}} \int_{0}^{1} \frac{\mathrm{I}+n^{3} x^{6}-\mathrm{I}}{\left(\mathrm{I}+n x^{2}\right) \sqrt{\left(\mathrm{I}-k^{2} x^{2}\right)\left(\mathrm{I}-x^{2}\right)}} d x\right] \\
& =\frac{8(A \alpha)^{2} w}{(A+a)^{2}} \mid \frac{\mathrm{I}}{n} F-\frac{\mathrm{I}}{n} \Pi+\frac{n\left(\mathrm{I}+k^{2}\right)+k^{2}}{n^{3}} \int_{0}^{1} \frac{\mathrm{I}-n x^{2}}{\sqrt{\left(\mathrm{I}-k^{2} x^{2}\right)\left(\mathrm{I}-x^{2}\right)}} d x \\
& \left.-\frac{\mathrm{I}-k^{2}}{n^{2}} \Pi+\frac{k^{2}}{n} \int_{0}^{1} \frac{x^{4} d x}{\sqrt{\left(\mathrm{I}-k^{2} x^{2}\right)\left(\mathrm{I}-x^{2}\right)}}\right\}
\end{aligned}
$$

Now

$$
\int_{0}^{1} \frac{\mathrm{I}-n x^{2}}{\sqrt{\left(1-k^{2} x^{2}\right)\left(1-x^{2}\right)}} d x=F+\frac{n}{k^{2}} E-\frac{n}{k^{2}} F
$$

$F, E, \Pi$ are the elliptic integrals of the first, second, and third kind.

It, therefore, remains only to evaluate the integral

$$
\int_{0}^{1} \frac{x^{4} d x}{\sqrt{\left(1-k^{2} x^{2}\right)\left(1-x^{2}\right)}}
$$

which can be done in the following manner:

$$
\begin{aligned}
& \frac{d}{d x}\left\{x \sqrt{\left(\mathrm{I}-k^{2} x^{2}\right)\left(\mathrm{I}-x^{2}\right)}\right\}=\left\{\frac{3 k^{2} x^{4}}{\sqrt{\left(\mathrm{I}-k^{2} x^{2}\right)\left(\mathrm{I}-x^{2}\right)}}\right. \\
& \left.-\frac{2\left(\mathrm{I}+k^{2}\right) x^{2}}{\sqrt{\left(\mathrm{I}-k^{2} x^{2}\right)\left(\mathrm{I}-x^{2}\right)}}+\frac{\mathrm{I}}{\sqrt{\left(\mathrm{I}-k^{2} x^{2}\right)\left(\mathrm{I}-x^{2}\right)}}\right\} d x \\
\because & \left.x \sqrt{\left(\mathrm{I}-k^{2} x^{2}\right)\left(\mathrm{I}-x^{2}\right)}\right]_{0}^{1}=\int_{0}^{1} \frac{3 k^{2} x^{4} d x}{\sqrt{\left(\mathrm{I}-k^{2} x^{2}\right)\left(\mathrm{I}-x^{2}\right)}} \\
- & \int_{0}^{1} \frac{2\left(\mathrm{I}+k^{2}\right) x^{2} d x}{\sqrt{\left(\mathrm{I}-k^{2} x^{2}\right)\left(\mathrm{I}-x^{2}\right)}}+\int_{0}^{1} \frac{d x}{\sqrt{\left(\mathrm{I}-k^{2} x^{2}\right)\left(\mathrm{I}-x^{2}\right)}}
\end{aligned}
$$


Hence

$$
\int_{0}^{1} \frac{x^{4} d x}{\sqrt{\left(1-k^{2} x^{2}\right)\left(\mathrm{I}-x^{2}\right)}}=\frac{-2\left(\mathrm{I}+k^{2}\right)}{3 k^{4}} E+\frac{2+k^{2}}{3 k^{4}} F
$$

Introducing the values from (9) and (Io) into equation (8), and collecting the terms containing $F, E$, and $\Pi$ separately, we shall finally get

$$
V=\frac{1}{6} \pi\left[F\left\{(A-a)^{2}+c^{2}-\frac{3\left(A^{2}-a^{2}\right)}{\tau v^{2}}\right\}+E\left\{2\left(A^{2}+a^{2}\right)-c^{2}\right\}-\frac{3 c^{2}(A-a)^{2}}{\tau v^{2}} \Pi\right.
$$

The elliptic integral of the third kind $\Pi$ can be transformed into the complete and incomplete elliptic integrals of the first and second kind, by the following relation: ${ }^{3}$

where

$$
\begin{aligned}
& \frac{k^{2} \sin \theta \cos \theta}{\sqrt{\left(\mathrm{I}-\sin ^{2} \theta\right)\left(\mathrm{I}-k^{2} \sin ^{2} \theta\right)}}\{\Pi-F\}= \\
& \frac{1}{2} \pi+F\left\{F\left(k^{\prime} \theta\right)-E\left(k^{\prime} \theta\right)\right\}-E F\left(k^{\prime} \theta\right)
\end{aligned}
$$

$$
k^{\prime 2}=\mathrm{I}-k^{2}
$$

$\theta$ is given by the relation

$$
n=-\mathrm{I}+k^{\prime 2} \sin ^{2} \theta
$$

$F\left(k^{\prime}, \theta\right)$ and $E\left(k^{\prime}, \theta\right)$ are the inconiplete elliptic integrals of modulus $k^{\prime}$ and anplitude $\theta$.

From equation $(\alpha)$ we get

$$
\sin ^{2} \theta=\frac{n+\mathrm{I}}{k^{\prime 2}}=\frac{\left(A^{2}-a^{2}\right)^{2}+c^{2}(A-a)^{2}}{\left(A^{2}-a^{2}\right)^{2}+c^{2}(A+a)^{2}}
$$

The value of $\Pi$ as obtained from equation (I 2) is given by the following equation:

$$
\Pi=F+\frac{(A+\alpha) \sqrt{ }(A+\alpha)^{2}+c^{2}}{c(\lambda-\alpha)}\left[\frac{\pi}{2}+F\left\{F\left(k^{\prime} \theta\right)-E\left(k^{\prime} \theta\right)\right\}-E F\left(k^{\prime} \theta\right)\right]
$$

\footnotetext{
${ }^{3} \lambda$. Cayley: Flementary Treatise on Filiptic Functions, \% I $S_{3}$.
} 
if now we introduce the value of $\Pi$ from the above equations into equation (I I) and simplifying, we shall finally get

$$
\begin{aligned}
V= & -\left(A^{2}-a^{2}\right) c\left[F\left\{F\left(k^{\prime} \theta\right)-E\left(k^{\prime} \theta\right)\right\}-E F\left(k^{\prime} \theta\right)\right] \\
& +\frac{c^{4}-\left(A^{2}-6 A a+a^{2}\right) c^{2}-2\left(A^{2}-a^{2}\right)^{2}}{3 \sqrt{(A+a)^{2}+c^{2}}} F+ \\
& \frac{2\left(A^{2}+a^{2}\right)-c^{2}}{3} \sqrt{(A+a)^{2}+c^{2}} E-c\left(A^{2}-a^{2}\right) \frac{\pi}{2}
\end{aligned}
$$

To recapitulate we have:

$$
M=4 \pi n n^{\prime}\left(V-V_{1}\right)
$$

$V$ is given by equation (I3), and $V_{1}$ is obtained from $V$ by replacing $c$ by $c_{1}$

$$
c=l+l_{1} \quad, \quad c_{1}=l-l_{1}
$$

$F$ and $E$ are the complete elliptic integrals of modulus $k$

$$
k^{2}=\frac{4 A \alpha}{(A+a)^{2}+c^{2}}
$$

$F\left(k^{\prime}, \theta\right)$ and $E\left(k^{\prime}, \theta\right)$ are the incomplete elliptic integrals of first and second kind of modulus $k^{\prime}$ and amplitude $\theta$

$$
\begin{aligned}
& k^{\prime 2}=I-k^{2} \\
& \sin ^{2} \theta=\frac{\left(A^{2}-a^{2}\right)^{2}+c^{2}(A-a)^{2}}{\left(A^{2}-a^{2}\right)^{2}+c^{2}(A+a)^{2}}
\end{aligned}
$$

Formula (I 3 ) is very similar in form to that given in Coffin's paper. The third term is exactly equal to the corresponding term in the Kirchhoff formula, and there is only a small difference in the first term. There is, however, a very great difference between the other two terms in Kirchhoff's formula. When the radii are equal the two formulæ will become identical, because the terms that involve the error then disappear. The test that Dr. Coffin applied to Kirchhoff's formula by deducing from it the self-inductance of a solenoid by making the radii equal is thus seen to be insufficient. 
The accuracy of formula ( $\mathrm{I}_{3}$ ) is shown by numerical tests given in another paper. ${ }^{4}$

For the case of two solenoids of the following dimensions:

$$
l=200, l_{1}=20 . \quad A=\mathrm{I} 5, a=\mathrm{IO}
$$

the value of $M$ given by formula (I 3 ) and confirmed by the formulæe of Roiti and of Searle and Airey is

$$
M=4 \pi m n^{\prime} \times 62 \mathrm{I} 3.4 .
$$

The value of the mutual inductances for the same case as calculated by Kirchhoff's formula as given in Coffin's paper gives the following:

$$
M=-4 \pi n n^{\prime} \times 21399
$$

This value is more than three times the true value of $M$. If we entirely neglect the correction factor due to the end effects, the mutual inductance is:

$$
M=4 \pi^{2} a^{2} l_{1} n n^{\prime}=4 \pi n n^{\prime} \times 6283 \cdot 19,
$$

a little more than I per cent greater than the true value of the mutual inductance.

The value of the self-inductance of solenoids can be easily obtained from equation (I 3 ), as was done by Coffin, by making $A=\alpha, l=l_{1}$. We thus obtain

$$
V=\frac{c^{4}+4 A^{2} c^{2}}{3 \sqrt{4 A^{2}+c^{2}}} F+\frac{4 A^{2}-c^{2}}{3} \sqrt{4 A^{2}+c^{2}} E
$$

To obtain $V_{1}$ we replace $c$ by $c_{1}$, but in this case

$$
\begin{gathered}
c_{1}=l-l_{1}=0 \\
\because V_{1}=\frac{8 A^{3}}{3} E
\end{gathered}
$$


Since, however, $k^{2}=\frac{4 A a}{(A+a)^{2}+c^{2}}=\mathrm{I}$ in this case

$$
\begin{aligned}
& \because E=\int_{0}^{\frac{\pi}{2}} \sqrt{\mathrm{I}-\sin ^{2} \phi} d \phi=\mathrm{I} \\
& \because V_{1}=\frac{8}{3} A^{3}
\end{aligned}
$$

and

$$
L=4 \pi n n^{\prime}\left\{\frac{c^{4}+4 A^{2} c^{2}}{3 \sqrt{4 A^{2}+c^{2}}} F+\frac{4 A^{2}-c^{2}}{3} \sqrt{4 A^{2}+c^{2}} E-\frac{8 A^{3}}{3}\right\}
$$

WASHIngTon, March 14, 1907. 